\title{
Appendix: Superfast Spreading Crust acronyms ${ }^{1}$
}

\author{
Expedition 335 Scientists $^{2}$
}

${ }^{1}$ Expedition 335 Scientists, 2012. Appendix: Superfast Spreading Crust acronyms. In Teagle, D.A.H., Ildefonse, B., Blum, P., and the Expedition 335 Scientists, Proc. IODP, 335: Tokyo (Integrated Ocean Drilling Program Management International, Inc.).

doi:10.2204/iodp.proc.335.105.2012

'Expedition 335 Scientists' addresses.
AARM: anisotropy of anhysteretic remanence

AF: alternating field

AMS: anisotropy of magnetic susceptibility

AMSOC: American Miscellaneous Society

APCT: advanced piston corer temperature tool

APS: Accelerator Porosity Sonde

ARM: anhysteretic remanent magnetization

BCR: bicenter reamer

BGRM: Bundesanstalt für Geowissenschafen und Rohstoffe magnetometer

BHA: bottom-hole assembly

BHTV: Borehole Televiewer tool

BOH: bottom of hole

BSJB: bit sub junk basket

CADA: cam-actuated drill-ahead

CDP: complex drilling proposal

ChRM: characteristic remanent magnetization

CORK: circulation obviation retrofit kit

CPF: crystal-plastic fabric

CRF: core reference frame

CSD: circular standard deviation

DC: drill collar

DC-SQUID: direct-current superconducting quantum interfer-

ence device

DESCINFO: descriptive and interpretive information

DI: deionized water

DIRM: drilling-induced remanent magnetization

DLL: Dual Laterolog

DP: dynamic positioning

DSDP: Deep Sea Drilling Project

DSI: Dipole Sonic Imager

EDTC: Enhanced Digital Telemetry Cartridge

EOP: end of pipe

EPR: East Pacific Rise

EXJB: external junk basket

FM: fishing magnet

FMS: Formation MicroScanner

FMS-sonic: Formation MicroScanner/Dipole Sonic Imager

FTJB: flow-through junk basket

FWHM: full width of half maximum

GAD: geocentric axial dipolar

GC: gas chromatography 
GPIT: General Purpose Inclinometry Tool

GPS: Global Positioning System

GRA: gamma ray attenuation

HLDS: Hostile Environment Litho-Density Sonde

HNGS: Hostile Environment Natural Gamma Ray Sonde

HRB: hard rock base

HRLA: High-Resolution Laterolog Array

IADC: International Association of Drilling Contractors

ICP-AES: inductively coupled plasma-atomic emission spectroscopy

IGRF: International Geomagnetic Reference Field

IODP: Integrated Ocean Drilling Program

IU: instrument units

IUGS: International Union of Geological Sciences

LDEO: Lamont-Doherty Earth Observatory

LDVT: linear voltage displacement transformer

LEH: logging equipment head

LEH-MT: Logging Equipment Head with Tension and Mud Temperature

LET: logarithm of extreme time

LIMS: Laboratory Information Management System

LOI: loss on ignition

LTD: low-temperature demagnetization

MAD: moisture and density

MADC: moisture and density, method $C$

MARK: Mid-Atlantic Ridge Kane Fracture Zone

MBR: mechanical bit release

mbrf: meters below rig floor

mbsf: meters below seafloor

m CSF-A: meters core depth below seafloor, method A

MCS: multichannel seismic

MORB: mid-ocean-ridge basalt

MRU: motion reference unit

MS: magnetic susceptibility

msb: meters subbasement

MSPOINT: point magnetic susceptibility

MTT: Modular Temperature Tool

NGR: natural gamma radiation

NGRL: Natural Gamma Radiation Logger

N-MORB: normal mid-ocean-ridge basalt

NOR: Geoset diamond core bit

NRM: natural remanent magnetization

OBH: ocean bottom hydrophone

ODL: Overseas Drilling Limited

ODP: Ocean Drilling Program

PCA: principal component analysis

PEF: photoelectric effect

POOH: pull out of hole
PPL: plane-polarized light

PSV: paleosecular variation

RBI: Rock Bit International

RCB: rotary core barrel

RCJB: reverse circulation junk basket

RFT: retrievable formation tester

RIH: run in hole

ROP: rate of penetration

ROV: remotely operated vehicle

RTD: resistance temperature detector

RSC: reflection spectroscopy color

SAM: special approximation method

SGT: Scintillation Gamma Ray Tool

SHIL: Section Half Imaging Logger

SHLF: section half

SHMSL: Section Half Multisensor Logger

SIF: subhorizontal irregular fracture

SPO: shape-preferred orientation

SRM: superconducting rock magnetometer

TAMU: Texas A\&M University

TAP: Temperature/Acceleration/Pressure tool

TCI: tungsten carbide inserts

TD: total depth

T/D: top drive

TP: total penetration

TS: thin section

UBI: Ultrasonic Borehole Imager

USIO: US Implementing Organization

UTC: Universal Time Coordinated

VCD: visual core description

VDS: vector difference sum

VIT: vibration-isolated television

VSI: Versatile Seismic Imager

VSP: vertical seismic profile

WHC: wireline heave compensator

WOB: weight on bit

WOW: waiting on weather

WR: whole round

WRMSL: Whole-Round Multisensor Logger

WST: Well Seismic Tool

WSTP: water-sampling temperature probe

WTR: Web Tabular Report

XPL: cross-polarized light

XRD: X-ray diffraction

Publication: 3 June 2012

MS 335-105 